\title{
Bacteriemia oculta en niños atendidos en el Complejo Asistencial Dr. Sótero del Río. Experiencia post vacuna neumocóccica conjugada
}

\author{
Isolda Budnik, Alejandra Sandoval, Alejandra Prado, Marcela Labbé, Anamaría Peña y Tamara Viviani
}

\author{
Occult bacteremia in Sótero del Río Hospital. \\ Experience after pneumococcal conjugated vaccine
}

Background: Occult bacteremia $(\mathrm{OB})$ is one of the possible diagnoses of children younger than 3 years with fever without source in the emergency room. Objective: describe OB in the era after introduction of pneumococcal vaccine in Chile. Patients and Methods: Prospective descriptive review of data of children with possible OB diagnosis, referred from the emergency department between 2010-2013. Results: Possible OB was diagnosed on 391 patients. 233 had focus, mainly respiratory virus and urinary tract infection. On 158 patients, probable BO was diagnosed, 20 had proven $\mathrm{OB}$ from which 15 had positive blood culture for Streptococcus pneumoniae. From these, 7 were fully or partially vaccinated. The serotype was identified on 14 cases: 6 were PCV10 vaccine serotypes (none of them vaccinated), 2 were serotype related (both partially vaccinated) and 6 were non vaccine serotypes (partially or totally vaccinated). Discussion: It is necessary to improve diagnostic methods for respiratory viruses and urinary tract infections and try to expand coverage of pneumococcal conjugated vaccines in the pediatric population in order to reduce the risk of invasive pneumococcal disease in Chile.

Key words: Occult bacteremia, Streptococcus pneumoniae, pneumococcal conjugated vaccine.

Palabras clave: Bacteriemia oculta, Streptococcus pneumoniae, vacuna neumocóccica conjugada.

\section{Introducción}

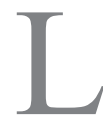

a fiebre es uno de los principales motivos de consulta de niños en el servicio de urgencia (SU) y corresponde aproximadamente a $20-30 \%$ de las atenciones ${ }^{1-5}$. Si bien, en la mayoría de los casos, se identifica el foco clínico causante del cuadro febril, hasta en $20 \%$ de los casos de niños bajo 36 meses de edad, esto no es posible ${ }^{4-6}$. En este grupo de pacientes, los síndromes febriles sin foco habitualmente corresponden a cuadros virales de escasa gravedad ${ }^{5-7}$, pero hasta en 10 a $25 \%$, la causa es una infección bacteriana invasora (IBI), como bacteriemia oculta (BO), infección osteo-articular (IOA), infección del tracto urinario (ITU) o meningitis bacteriana aguda $(\mathrm{MBA})^{3,5,7}$. De las IBI, la BO es la más frecuente, alcanzando hasta $75 \%$ en este grupo etario ${ }^{1} \mathrm{y}$ por esta razón, cuando en la evaluación inicial no se logra encontrar foco, se debe iniciar estudio del paciente como sospecha de BO.

Previo a la introducción de las vacunas conjugadas, los microorganismos de mayor importancia causantes de IBI eran Haemophilus influenzae serotipo b (Hib) y Streptococcus pneumoniae ${ }^{1}$. Históricamente Hib fue una importante causa de morbimortalidad en este grupo etario; sin embargo, después de la introducción universal de la vacuna conjugada, esta etiología prácticamente ha desaparecido ${ }^{1}$. En concomitancia con la disminución de enfermedad invasora por Hib, se detectó un aumento de la enfermedad invasora causada por $S$. pneumoniae, con una importante carga de enfermedad a lo largo del mundo, transformándose en la etiología más frecuente de BO en lactantes; se, aísla en 80-90\% de los hemocultivos con crecimiento bacteriano y alcanza una mortalidad de $1,4 \%{ }^{1,8,9}$. Debido a la carga de enfermedad neumocóccica y a su gravedad, se desarrollaron vacunas anti-neumocóccicas, en base a polisacáridos y conjugadas (PCVs), para incorporar a los programas de inmunización. La primera vacuna conjugada disponible internacionalmente fue la 7-valente (PCV7), que contiene a los serotipos 4, 6B, 9V, 14, 18C, $19 \mathrm{~F}$ y $23 \mathrm{~F}^{8,10}$. Posteriormente se desarrollaron vacunas con mayor número de serotipos, la PCV 10-valente (PCV10), que agregó a los serotipos 1, 5 y 7F y la PCV13-valente (PCV13), que además de los mencionados sumó los serotipos 3, 6A, 19A $\mathrm{A}^{8,10-12}$. Los serotipos 6A y 19A corresponden a los denominados serotipos relacionados a PCV10, los que a pesar de no estar incluidos en la vacuna, alcanzarían un grado de protección cruzada a través de los serotipos $6 \mathrm{~B}$ y $19 \mathrm{~F}$, respectivamente ${ }^{13.14}$.
Complejo Asistencial Dr. Sótero del Río, Santiago, Chile.

Unidad de Infectología Pediátrica. (IB, AS, AP, TV).

Pontificia Universidad Católica de Chile.

Facultad de Medicina, Servicio de Pediatría (ML)

Departamento de Enfermedades Infecciosas e Inmunología Pediátrica (AP).

Establecimiento(s) donde se realizó el trabajo: Complejo Asistencial Dr. Sótero del Río.

Los autores no tenemos conflictos de interés.

Fuente de Financiamiento: no hubo.

Recibido: 19 de octubre de 2016 Aceptado: 30 de marzo de 2017

Correspondencia a: Isolda Budnik Ojeda isolbudnik@gmail.com 
En el contexto epidemiológico actual, luego de la introducción de vacunas conjugadas, es importante destacar la notable disminución de las IBI en los últimos años, especialmente MBA y BO. En Estados Unidos de América (E.U.A.) se describen reducciones de incidencia de MBA significativas, de hasta $30 \%$, siendo en lactantes de $64 \%$ para todas las causas y de $93 \%$ para los serotipos vaccinales incluidos en $\mathrm{PCV}^{8}$. Para $\mathrm{BO}$, se vio reducción de $84 \%$ de las bacteriemias por $S$. pneumoniae entre los 3 meses y los 3 años $^{8}$. En Chile, el ISP ha reportado disminución de las cepas confirmadas de S. pneumoniae, causantes de enfermedad neumocóccica invasora (ENI), de $65 \%$ aproximadamente, entre 2007 y 2014 en niños bajo 2 años de edad.

La incidencia actual de bacteriemia reportada en la literatura médica es de $1,5-2 \%{ }^{6,15}$ en los pacientes bajo 36 meses de edad con síndrome febril sin foco (SFSF) $\mathrm{y}$ buen aspecto general. Estudios nacionales revelan una incidencia similar $(1,2 \%)^{3,17}$, y otros internacionales, tasas de morbimortalidad de $0,3 \%{ }^{15,16}$, lo que refleja un buen pronóstico para estos pacientes. Un estudio nacional estimó una mortalidad anual potencial por ENI de 12.00028.000 niños. En este escenario, la PCV podría salvar una vida por cada 1.100 niños vacunados y evitar un caso de enfermedad invasora por cada 13 niños vacunados ${ }^{18}$.

En Chile se implementó la incorporación de PCV10, en noviembre del 2010, sin catch up, con un esquema $3+1$ (2-4-6 meses + refuerzo a los 12 meses), comenzando con la vacunación de niños de 2 meses de edad a enero de 2011. Por datos de experiencias internacionales, en enero de 2012 se cambió el esquema a 2+1 (2-4 meses + refuerzo a los 12 meses $^{10,12}$. Desde febrero de 2016, en la Región Metropolitana se está vacunando con PCV13 con esquema $2+1$ a los 2-4 y $12 \mathrm{~m}$ y desde julio de 2016 con esquema $3+1$ a los 2-4-6 y $18 \mathrm{~m}$.

Al considerar la nueva situación epidemiológica en Chile y que las infecciones virales siguen siendo la etiología más frecuente de fiebre en un niño, la probabilidad de BO es cada vez menor. Por esta razón, la norma de sospecha y manejo del paciente bajo 3 años de edad y con fiebre sin foco debiera adecuarse a esta nueva realidad.

El presente estudio se realizó en el Complejo Asistencial Dr. Sótero del Río (CASR), centro terciario que atiende a los beneficiarios del área sur-oriente de Santiago y tiene una población asignada de 350.000 niños/adolescentes bajo 15 años de edad.

El objetivo principal es describir y evaluar el diagnóstico de BO en niños bajo 36 meses que consultan al SU por fiebre sin foco, que son catalogados como $\mathrm{BO}$ posible y derivados a nuestra unidad (Unidad de Infectología pediátrica del CASR) y como objetivos secundarios describir los microorganismos que con mayor frecuencia causan BO en este grupo etario, teniendo especialmente en consideración $S$. pneumoniae y sus serotipos.

\section{Metodología}

Se realizó un estudio descriptivo, prospectivo, en el período diciembre de 2010-diciembre de 2013, que incluyó a todos los niños entre 6 semanas y 36 meses, derivados desde SU a nuestra unidad, con diagnóstico de $\mathrm{BO}$ posible. Se contó con la aprobación del Comité de Ética.

Se establecieron las siguientes definiciones:

- BO posible: fiebre $\leq$ a $72 \mathrm{~h}$ de evolución, sin foco al examen físico ni por exámenes básicos (sedimento orina, rx tórax, etc.) más $\mathrm{PCR} \geq$ a $90 \mathrm{mg} / \mathrm{L}$ y/o leucocitosis $\geq \mathrm{a} 15.000 / \mathrm{mm}^{3}$ y que contaran con hemocultivo tomado.

- BO probable: BO posible, en los que no se identificó foco tras el seguimiento clínico ni por exámenes de laboratorio.

- BO probada: BO probable, con hemocultivo positivo (se excluyeron contaminantes).

Este estudio no incluyó intervención alguna adicional a la normativa local de estudio y tratamiento de BO.

Durante la primera consulta en la Unidad de Infectología se realizó un examen físico acucioso, se tomaron exámenes de acuerdo a los hallazgos y se llenó la ficha del protocolo. Se efectuó seguimiento clínico diario hasta la desaparición de la fiebre y control posterior al término del tratamiento antimicrobiano.

Se recolectó información relevante que incluyó: datos epidemiológicos (género, edad), antecedentes de vacunación (PNI, PCV, número de dosis de PCV), síntomas y signos, días de fiebre, temperatura máxima, aspecto general al momento de la consulta, exámenes de laboratorio (leucocitos, proteína $\mathrm{C}$ reactiva (PCR), sedimento de orina, panel viral respiratorio, urocultivo, hemocultivo, citoquímico y cultivo de líquido cefalorraquídeo). Se efectuaron otros exámenes de acuerdo a los hallazgos. El panel viral respiratorio se realizó mediante inmunofluorescencia directa (IFD) e incluye la identificación de virus respiratorio sincicial (VRS), virus parainfluenza 1,2 y 3, adenovirus (ADV) y metapneumovirus; la muestra de orina para sedimento y cultivo fue tomada por cateterismo vesical y se sospechó ITU si el sedimento de orina centrifugada informaba más de 5 leucocitos por campo y definitiva cuando hubo un recuento mayor a $10^{4} \mathrm{ufc} / \mathrm{ml}$; los hemocultivos fueron procesados en método automatizado BacT/Alert ${ }^{\circledR}$. Todos los hemocultivos con crecimiento de S. pneumoniae fueron enviados al Instituto de Salud Pública (ISP) para su serotipificación y estudio de resistencia. La serotipificación capsular se realiza de acuerdo a estándares y control de calidad de la red SIREVA-OPS/OMS y desde el año 2015 se encuentran disponibles métodos moleculares ${ }^{12}$. La susceptibilidad antimicrobiana se realizó mediante el método de difusión en agar y la determinación de la concentración inhibitoria 
mínima (CIM) a penicilina y cefotaxima, utilizando los puntos de corte establecidos por el CLSI y control de calidad de la red SIREVA-OPS/OMS ${ }^{12}$.

Según estado de vacunación, se definió como:

- No vacunados: con menos de una dosis de PCV

- Parcialmente vacunados: con una o dos dosis de PCV

- Totalmente vacunados: con al menos tres dosis de PCV

Para evaluar la protección vaccinal, se definieron tres grupos según el número de dosis de PCV recibidas:

- Grupo protegido: recibió tres dosis de PCV10

- Grupo con protección parcial: recibió dos dosis de PCV 10

- Grupo no protegido: recibió una dosis de PCV 10 o ninguna.

De acuerdo a la normativa de la Unidad de Infectología, el antimicrobiano indicado frente a la sospecha de BO es ceftriaxona intravenosa en dosis de $50 \mathrm{mg} / \mathrm{kg}$ cada $24 \mathrm{~h}$ y la administración de al menos una dosis se consideró tratamiento.

Se efectuó análisis estadístico descriptivo con medidas de concentración y frecuencias en el programa Epi Info versión 3.4.1. Se realizaron pruebas de chi cuadrado y se consideró como significativos valores de $\mathrm{p} \leq 0,05$.

\section{Resultados}

Durante el período de estudio fueron derivados, desde el SU a nuestra unidad 625 niños con diagnóstico de BO posible. De ellos, $37 \%$, fueron excluidos por no cumplir con los criterios establecidos de BO posible. Un total de 391 pacientes sí cumplieron los criterios y es este grupo el evaluado para los fines del presente estudio. La descripción se detalla en el flujograma (Figura 1).

Se encontró foco infeccioso en 233 pacientes $(59,6 \%)$ que se consideró causa del cuadro febril; los diagnósticos finales más frecuentes fueron: infección respiratoria viral (65,2\%) e ITU (21,5\%). En $124(81,6 \%)$ pacientes con sospecha de infección respiratoria viral se tomó panel para IFD. Se identificó al menos un virus respiratorio en $83(54,6 \%)$ pacientes y en más de $2 / 3$ de aquellos sin identificación viral se diagnosticó infección respiratoria alta por clínica y evolución auto-limitada. De las 83 IFD positivas, 73,5\% correspondió a detección de ADV. En todos los pacientes con diagnóstico definitivo de ITU, es decir, con urocultivo positivo, el sedimento de orina tomado en la primera consulta en SU, fue normal y por lo tanto el diagnóstico de referencia a nuestra unidad fue BO posible.

Habiendo descartado los síndromes febriles con foco identificado durante la evolución de las atenciones médicas, el grupo definitivo de niños que cumplió los
- Pacientes con diagnóstico de BO posible derivados desde el S.U.

- Pacientes excluidos por no cumplir con los criterios establecidos de BO posible*

- Pacientes que cumplieron los criterios de BO posible

- Pacientes en los que se encontró foco infeccioso durante seguimiento**

- Pacientes que cumplieron los criterios de BO probable

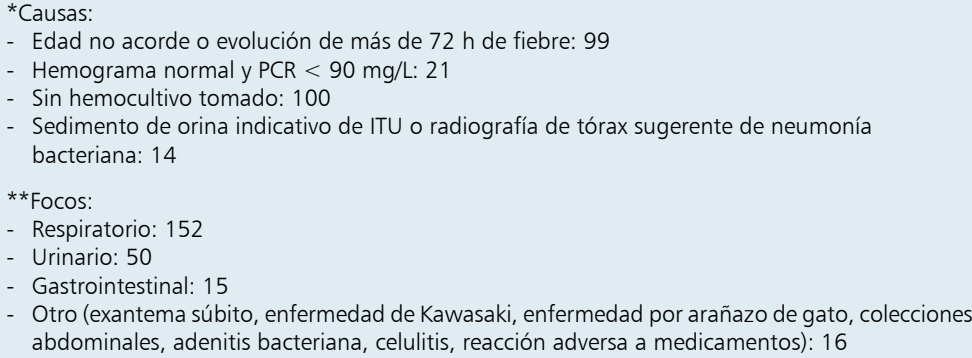

Figura 1. Flujograma.

criterios de BO probable fue de 158 (40,4\% del grupo inicial que cumplían los criterios de $\mathrm{BO}$ posible). De ellos, en 20 (12,6\%), hubo crecimiento bacteriano en el hemocultivo, es decir, correspondieron a BO probada. El microorganismo más frecuente fue $S$. pneumoniae, en 15 pacientes (75\%). En la Tabla 1 se describen los microorganismos aislados. La mayoría de los hemocultivos positivos $(85 \%)$ correspondieron a pacientes bajo 2 años. Del mismo modo, de los hemocultivos positivos para $S$. pneumoniae, $80 \%$ de los pacientes era menores de 2 años.

Al considerar la protección con vacuna neumocócica conjugada, de los 158 pacientes con BO probable,

Tabla 1. Microorganismos aislados en hemocultivos de pacientes con BO definitiva

\begin{tabular}{lcc}
\hline Hemocultivos positivos & $\mathbf{n}$ & $\mathbf{( \% )}$ \\
$\mathbf{n}=\mathbf{2 0}$ & $(\mathbf{1 0 0 )}$ \\
\hline Streptococcus pneumoniae & 15 & $(75,0)$ \\
Klebsiella pneumoniae & 2 & $(10)$ \\
\hline Enterococcus faecalis & 1 & $(5,0)$ \\
Neisseria meningitidis & 1 & $(5,0)$ \\
Salmonella enterica serovar Typhi & 1 & $(5,0)$ \\
\hline
\end{tabular}




\begin{tabular}{|c|c|c|c|}
\hline $\begin{array}{l}\text { Serotipo } \\
(n=15)\end{array}$ & $\begin{array}{l}\mathrm{n} \text { de dosis } \\
\text { VPC }\end{array}$ & $\begin{array}{c}\text { Serotipo vaccinal } \\
\text { VPC10 }\end{array}$ & $\begin{array}{c}\text { Serotipo vaccinal } \\
\text { VPC13 }\end{array}$ \\
\hline 3 & 2 & No & Sí \\
\hline $6 \mathrm{~A}$ & 2 & No & Sí \\
\hline 14 & 0 & Sí & Sí \\
\hline 14 & 0 & Sí & Sí \\
\hline 14 & 0 & Sí & Sí \\
\hline 14 & 0 & Sí & Sí \\
\hline 14 & 0 & Sí & Sí \\
\hline $15 F$ & 2 & No & No \\
\hline $18 \mathrm{C}$ & 0 & Sí & Sí \\
\hline $19 A$ & 2 & No & Sí \\
\hline $24 \mathrm{~F}$ & 3 & No & No \\
\hline $25 A$ & 4 & No & No \\
\hline 27 & 0 & No & No \\
\hline 38 & 4 & No & No \\
\hline No identificado & 0 & - & - \\
\hline
\end{tabular}

$102(64,6 \%)$ estaban total o parcialmente protegidos y $56(35,4 \%)$ no protegidos. De los 15 pacientes con diagnóstico de $\mathrm{BO}$ por $S$. pneumoniae, 7 estaban total o parcialmente protegidos con vacuna conjugada y 8 no estaban protegidos. No hubo diferencias estadísticamente significativas al comparar los pacientes con bacteriemia por $S$. pneumoniae que recibieron $\geq 3$ dosis de vacuna en comparación con aquellos que recibieron $\leq 2$ dosis ( $p \leq 0,05)$, como tampoco en aquellos que recibieron $\geq 2$ dosis de vacuna en comparación con aquellos que recibieron $\leq 1$ dosis $(\mathrm{p} \leq 0,05)$.

De los 15 casos con $S$. pneumoniae aislado en hemocultivo, se logró identificación de serotipo en 14. De ellos: seis correspondieron a serotipos contenidos en PCV10 (y ocurrieron en niños no vacunados), dos a serotipos relacionados a PCV10 (ambos niños parcialmente protegidos) y seis a serotipos no vaccinales (en niños parcial o totalmente vacunados, de quienes $5(83,3 \%)$ tenían al menos dos dosis y $3(50 \%)$ tenían al menos tres dosis de PCV10). La Tabla 2 muestra los serotipos identificados y el número de dosis de PCV10 para cada paciente.

\section{Discusión}

La fiebre es uno de los motivos más frecuentes de consulta en el SU en niños bajo 36 meses. Si el paciente no tiene foco y está en buenas condiciones generales, el problema es decidir, en la atención de urgencia, qué conducta tomar, teniendo en cuenta que la mayoría de los cuadros corresponden a una infección viral auto-limitada $\mathrm{y}$ de buen pronóstico, en menor número a ITU y un grupo pequeño de estos pacientes podría tener una IBI. Por esta razón, se han desarrollado múltiples guías de manejo de niño bajo 3 años con fiebre sin foco, con el objetivo de seleccionar a aquellos pacientes a los que estudiaremos, ahorrar tiempo y recursos y focalizar todo el esfuerzo en identificar niños de alto riesgo de IBI.

En E.U.A., previo a la vacunación anti-neumocóccica, la incidencia anual de BO era de aproximadamente 240/100.000 casos, siendo $S$. pneumoniae, el microorganismo más frecuentemente aislado ${ }^{8}$. Estudios post vacunación con PCVs, han demostrado una dramática caída de ENI y por lo tanto de la entidad $\mathrm{BO}^{4,7}$. Considerando este nuevo escenario epidemiológico, la posibilidad de BO en un niño bajo 3 años de edad, con fiebre de pocos días de evolución, buen aspecto general y sin foco, es cada vez menor ${ }^{16}$. En la literatura médica se describe que aproximadamente $1-3 \%$ de estos niños, con fiebre sin foco, tendrá en definitiva una $\mathrm{BO}^{3}$. En nuestra serie los resultados muestran una cifra levemente más alta, de $3,8 \%$, probablemente explicada por el uso de normas con criterios de diagnóstico para $\mathrm{BO}$ posible, probable y probada.

En nuestro estudio, del grupo inicialmente derivado a la Unidad de Infectología desde el SU como BO posible, $37,4 \%$ no cumplía con los criterios definidos, lo que indica que se requiere capacitar al equipo del SU, para lograr una mayor adhesión a la norma de manejo del "menor de 3 años con fiebre sin foco". De las BO posibles, finalmente $40,4 \%$ correspondió a BO probable, según los criterios del estudio, lo que refuerza una vez más, la importancia de un acabado examen físico al momento de buscar el foco de la fiebre y el seguimiento de estos pacientes. Fue posible diagnosticar la causa de la fiebre, ya fuese por clínica y/o laboratorio, en más de la mitad de los pacientes que iniciaron el estudio de BO posible en la Unidad de Infectología, siendo las etiologías más frecuentes las infecciones respiratorias virales en $65,2 \%$, ITU en $12,8 \%$ y cuadros gastrointestinales en 3,8\%. Otros diagnósticos menos frecuentes fueron: exantema súbito, enfermedad de Kawasaki, infección de piel y tejidos blandos, enfermedad por arañazo de gato, adenitis cervical aguda, apendicitis con colecciones intrabdominales y reacciones adversas a medicamentos. No hubo casos de infecciones del SNC ni IOA. Es necesario destacar que un alto porcentaje de los pacientes que se diagnosticaron y trataron inicialmente como BO posible, correspondieron a ITU con sedimento de orina normal e infecciones respiratorias virales, siendo el ADV el agente etiológico más frecuente. Ambas condiciones pueden producir elevación marcada de parámetros inflamatorios, lo cual induce a pensar en IBI. 
Respecto al diagnóstico de infección respiratoria viral, es importante mencionar que el examen se realizó en pacientes sin sintomatología respiratoria evidente y eso explica, probablemente, el alto número de pacientes con IFD positiva para ADV, causante a veces, de cuadros únicamente febriles. Existe bastante evidencia en la literatura médica, que demuestra la sensibilidad y especificidad de los métodos moleculares muy por sobre la IFD (88 y $98 \%$ vs 68 y 70\%, respectivamente) $)^{19-25}$. Gharabaghi y cols., compararon el rendimiento de cuatro ensayos comerciales de reacción de polimerasa en cadena-RPC con IFD y aislamiento viral en 750 hisopados nasofaríngeos de pacientes pediátricos, obteniendo un porcentaje de positividad adicional de $28,5 \%$, lo que se traduce en un incremento de la sensibilidad de detección de virus respiratorios en niños, de $74,3 \%$ sobre la IFD y el cultivo viral $^{20}$. Particularmente para ADV, la sensibilidad de los métodos moleculares es de $78-100 \%$ mientras que la de IFD es de $50-70 \%{ }^{21,23-25}$. Con estos datos, es posible suponer que un número considerable de pacientes que quedan catalogados como BO probable, correspondan a infecciones por virus respiratorios no diagnosticados por IFD, especialmente ADV.

Por otra parte, si consideramos el alto número de pacientes diagnosticados con ITU por urocultivo, cuyos sedimentos urinarios fueron normales, significa que no es posible excluir este diagnóstico en pacientes bajo de 3 años de edad con fiebre inicial sin foco y que probablemente sería interesante evaluar técnicas rápidas que sugieran $\mathrm{ITU}^{26}$.

La mayoría de los hemocultivos positivos (85\%) correspondieron a lactantes, lo que coincide con los resultados reportados por Ishmin y cols. ${ }^{1}$. El microorganismo recuperado con mayor frecuencia desde hemocultivos de niños con BO probable fue $S$. pneumoniae y como era de esperar, fue menos frecuente, aunque estadísticamente no significativo, en el grupo de niños protegidos. Estudios como el de Kaiser Permanente describe el gran impacto de la PCV7 en niños entre 3 y 36 meses ambulatorios con bacteriemia por $S$. pneumoniae, con marcado descenso de su incidencia en Carolina del Norte ${ }^{8-27}$. En nuestro estudio es destacable que los pacientes protegidos y parcialmente protegidos, presentaron ENI por serotipos no vaccinales, mientras que los no protegidos tuvieron infecciones tanto por serotipos vaccinales como no vaccinales. Todos los casos de bacteriemia por $S$. pneumoniae de los serotipos contenidos en la PCV10 fueron en pacientes no vacunados, los casos por serotipos relacionados a PCV10 estaban parcialmente vacunados y los casos por serotipos no contenidos en la PCV10 habían recibido, en prácticamente todos los casos, al menos dos dosis de vacuna. Esto hace sentido, ya que lo esperable es que dos dosis sean suficientes para obtener una buena protección contra los serotipos vaccinales contenidos en la PCV10, pero que probablemente dos dosis fueran insuficientes para lograr una adecuada protección cruzada contra los serotipos 6A y 19A. Para los niños protegidos, lo esperado es tener prácticamente sólo casos por serotipos no vaccinales, lo que concuerda con lo encontrado en nuestro estudio. Carstairs y cols., reportaron 0 versus $2,4 \%$ de hemocultivos positivos para S. pneumoniae en niños bajo 36 meses de edad, con fiebre, evaluados en el S.U. y que habían recibido al menos una dosis de PCV7 comparado con aquellos que no estaban inmunizados ${ }^{8-28}$. Si bien en nuestra experiencia, encontramos casos de bacteriemia por S. pneumoniae, tanto en pacientes vacunados como en no vacunados, sólo se identificaron serotipos no vaccinales en los pacientes vacunados con al menos una dosis, lo que es comparable con este último estudio.

Desde hace varios años, tras la incorporación de la PCV7 al programa nacional de inmunizaciones en algunos países, se ha observado el fenómeno de reemplazo de serotipos vaccinales, definido como la disminución de la prevalencia de serotipos de $S$. pneumoniae incluidos en la vacuna, con un concomitante incremento de serotipos no vaccinales ${ }^{8}$. Reportes en la literatura científica muestran que el mayor reemplazo de serotipos en ENI está dado por el aumento de los serotipos 19A, 1, 5, 7F, 15 y $33 \mathrm{~F}$, siendo el 19A predominante en varios países incluyendo Chile y E.U.A. ${ }^{9,29-31}$. Se infiere que, además del impacto positivo causado por la PCV7, que genera un aumento del serotipo 19A, el uso indiscriminado de antimicrobianos, especialmente de macrólidos, sería un factor contribuyente de este fenómeno, especialmente de las cepas de 19A resistentes a antimicrobianos ${ }^{8,32}$. Se ha demostrado que los serotipos de reemplazo en los niños bajo 5 años, coinciden con los de los adultos mayores de 65 años, lo que favorece la incorporación de otras vacunas que incluyen a más serotipos invasores ${ }^{8}$.

Las nuevas PCVs, 10 y 13 valentes, han logrado coberturas de 90 y $92 \%$ de los serotipos responsables de la mayor carga de ENI en niños bajo 5 años en Europa y E.U.A., respectivamente ${ }^{8,32}$, con efectividad por efecto rebaño en personas mayores de 65 años. Los serotipos de S. pneumoniae con mayor resistencia antimicrobiana son 6A, 6B, 9V, 14, 19A, 19F y 23F, dos (6A y 19A), no están incluidos en la PCV7 ni PCV10. La PCV13 suma los serotipos 3, 6A y $19 \mathrm{~A}^{8,10}$. Un estudio publicado por Pilishvili y cols., mostró que los serotipos adicionales contenidos en la PCV13, dieron cuenta de $63 \%$ de las ENI en niños bajo 5 años en E.U.A., entre los años 2006 y $2007^{30}$. El grupo de Dagan, en Beer-Sheva, Israel, evaluó el potencial de cobertura de PCV7, PCV10 y PCV13 contra la ENI, OMA, conjuntivitis aguda y portación nasofaríngea (NF) en el sur de Israel, donde no se había introducido aún la PCV7. En ese estudio se demostró que la cobertura para ENI para los serotipos contenidos en la PCV7 fue de 44\%; para PCV10 fue de $68 \%$ (y considerando la protección 
cruzada de $6 \mathrm{~B}$ con $6 \mathrm{~A}$, la cobertura ascendía a 74\%); y para PCV13 mostró la mayor cobertura llegando hasta $84 \%{ }^{33}$. Además, en este estudio se calculó y se analizó el potencial de cobertura de las PCVs en forma separada para cepas con resistencia antimicrobiana asociadas a ENI y otitis media aguda (OMA), informando que PCV13 podría prevenir más de $90 \%$ de las ENI producidas por cepas resistentes a penicilinas, macrólidos y multiresistentes ${ }^{33}$. Considerando el incremento mundial del serotipo 19A y ser el mayor causante de ENI por serotipos de reemplazo, parece ser fundamental que la vacuna incorporada a los programas nacionales de inmunización de cada país incluya este serotipo.

En Chile, luego de la introducción de PCV 10, se observa un descenso general de la tasa de incidencia anual de las cepas confirmadas por 100.000 habitantes (desde 5,32 en 2009 hasta 3,71 en 2014) ${ }^{12}$, datos confirmados por el laboratorio de referencia ISP. Datos de la misma fuente dan cuenta de un descenso de ENI en $66,2 \%$, entre el 2007 y el 2014, en el grupo etario menor de 1 año ${ }^{12}$. En el mismo período, el grupo etario entre 12 y 23 meses, mostró un descenso similar de ENI en 64,4\%. A diferencia de éstos, el grupo de adultos mayor de 65 años mostró un aumento de ENI de 23,4\%, en el período comprendido entre 2010 y 2014. Es necesario poner atención a este fenómeno, ya que los adultos mayores de 65 años son un grupo vulnerable, al igual que los niños y debieran incluirse como grupo especial de protección de ENI a modo de disminuir su morbimortalidad, hospitalizaciones y costos, tanto personales como económicos, derivados de las infecciones causadas por este agente.

Estudios han demostrado una relación directa entre la portación nasofaríngea (NF) y la $\mathrm{ENI}^{34}$. Se ha demostrado que las PCVs reducen tanto la adquisición NF de $S$. pneumoniae como la ENI, en personas vacunadas (efecto directo), como también en personas no vacunadas (efecto indirecto $)^{34}$. Si bien se ha demostrado ampliamente el efecto directo de todas las PCVs, hasta el momento los estudios con PCV7 y PCV13 han demostrado claros resultados respecto a la reducción de esta portación, impactando así en el efecto indirecto esperado ${ }^{35-36}$. Esto se hace más evidente con la introducción de la vacuna PCV13, la que presenta contundente evidencia de protección de rebaño para los serotipos adicionales incorporados en esta vacuna $^{37-40}$. Experiencias como las de Inglaterra y Gales han presentado resultados de portación, tras dos años de introducida la vacuna PCV13, en los que se ha demostrado un completo reemplazo de serotipos NF aislados previos al uso de la vacuna, con predominio de serotipos menos invasores y con menor riesgo de causar $\mathrm{ENI}^{37}$. Sin embargo, otros estudios como el COMPAS, que incluyó más de 23.000 niños en Latinoamérica, si bien demostró reducción de neumonías adquiridas en la comunidad, de OMAs confirmadas (totales y específicamente por serotipos vaccinales) y de ENI (totales y específicamente por serotipos vaccinales), no demostró reducción de la portación NF, factor determinante de la inmunidad de rebaño ${ }^{41}$. Por otra parte, tanto en Chile como en otras latitudes, se ha registrado incremento de los serotipos no vaccinales, especialmente del 19A. Reportes del ISP demuestran un aumento mantenido desde $3 \%$ en 2007 a $10 \%$ en 2015 , en la población general y particularmente desde $5 \%$ en 2007 a $23 \%$ en 2015 , en niños bajo 2 años ${ }^{12}$. Es así, como al elegir la vacuna que formará parte del PNI de nuestro país, será fundamental, por una parte, el impacto que ésta tenga sobre la portación NF y por ende en el efecto indirecto o inmunidad de rebaño, así como también, la mayor cobertura para serotipos causantes de ENI (incluidos los de reemplazo), especialmente en la población adulta mayor y pediátrica no vacunada, con mayor riesgo de $\mathrm{ENI}^{34,42,43}$.

\section{Conclusiones}

La experiencia con este estudio nos muestra que, a pesar de existir una norma clara de "manejo de niño menor de 36 meses, febril y sin foco", con el fin de focalizar el estudio y tratamiento en el grupo de pacientes con riesgo de IBI, se diagnostican erróneamente muchos pacientes como sospecha de BO. En más de la mitad de estos casos, ocurre en niños con ITU e infección por ADV, que suelen presentar elevación marcada de parámetros inflamatorios y demora de $24-48 \mathrm{~h}$ en el informe definitivo del urocultivo. En este grupo de pacientes, es fundamental el control evolutivo, tanto clínico como de laboratorio y se hace necesario mejorar la detección de virus respiratorios, especialmente de $\mathrm{ADV}$, con métodos más sensibles (RPC). Por otra parte, hay que hacer hincapié en la necesidad de tomar correctamente el urocultivo (por sondeo cuando corresponda) y esperar su resultado, ya que un sedimento de orina normal no descarta la posibilidad de ITU. Teniendo en cuenta los resultados del presente estudio, sería pertinente actualizar la guía de manejo de síndrome febril en el SU, agregar a esta norma nuevos métodos de detección de ADV y revisar la sensibilidad de otros métodos de sospecha de ITU.

Respecto a los microorganismos causantes de bacteriemia, en este grupo de pacientes, a pesar del nuevo escenario epidemiológico en el que nos encontramos tras la incorporación de las PCV, S. pneumoniae sigue siendo el microorganismo con mayor frecuencia aislado en los hemocultivos, por cobertura vaccinal incompleta o por serotipos no vaccinales. Es necesario recalcar la importancia de la PCV y su cobertura, mantener los sistemas de vigilancia para detectar las variaciones de la epidemiología, tanto nacional como mundial, y ser activos para considerar ajustes en nuestro PNI, evaluando 
su costo efectividad y determinando el verdadero impacto que ésta produciría en nuestra realidad, estableciendo el tipo de esquema a utilizar, incluyendo PCVs con la mayor cobertura de serotipos de $S$. pneumoniae, y en especial que incluyan al serotipo 19A y que disminuyan la portación NF, logrando un efecto rebaño y un real impacto en salud pública.

\section{Resumen}

Introducción: La bacteriemia oculta (BO) es uno de los diagnósticos que se plantean en los niños bajo 3 años de edad que se presentan con fiebre sin foco en el servicio de urgencia. Objetivo: Describir el diagnóstico de BO luego de la introducción de la vacunación universal para Streptococcus pneumoniae en Chile. Materiales y Métodos: Revisión descriptiva de seguimiento prospectivo de datos de niños con diagnóstico de BO posible derivados del SU entre 2010 y 2013. Resultados: Se diagnosticó BO posible en 391 pacientes. En 233 pacientes se encontró foco, siendo infecciones respiratorias virales e infección urinaria las más frecuentes. En 158 pacientes se diagnosticó BO probable, en 20 BO probada y 15 tuvieron hemocultivos positivos para $S$. pneumoniae. De estos últimos 7 estaban total o parcialmente vacunados. Se identificó serotipo en 14 casos: 6 serotipos vaccinales incluidos en PCV10 (ninguno vacunado), 2 serotipos relacionados (ambos parcialmente protegido) y 6 serotipos no vaccinales (parcial o totalmente vacunados). Discusión: Es necesario mejorar las técnicas diagnósticas de infecciones respiratorias virales e infección urinaria e intentar ampliar la cobertura de las vacunas neumocóccicas conjugadas en la población pediátrica, para reducir el riesgo de enfermedades neumocóccicas invasoras en Chile.

\section{Referencias bibliográficas}

1.- Ishimine P. Fever without source in children 0 to 36 months of age. Pediatr Clin North Am 2006; 53: 167-94.

2.- Arora R, Mahajan P. Evaluation of child with fever without source: review of literature and update. Pediatr Clin North Am 2013; 60: 1049-62.

3.- Brockmann P, Ibarra X, Silva I, Hirsch T. Etiología del síndrome febril agudo sin foco en niños bajo 36 meses de edad que consultan a un servicio de urgencia. Rev Chilena Infectol 2007; 24: 33-9.

4.- Marcondes B, Morais D, de Paulis M, de Ulhôa A, Elias A. Fever without source: evaluation of a guideline. J Pediatr (Rio J). 2009; 85: 426-32.

5.- Pulliam P, Attia M, Cronan K. C-reactive protein in febrile children 1 to 36 months of age with clinically undetectable serious bacterial infection. Pediatrics 2001; 108: 1275-9.

6.- Lee G, Harper M. Risk of bacteremia for febrile young children in the post-Haemophilus influenza type b era. Arch Pediatr Adolesc Med 1998; 152: 624-8.

7.- Finkelstein J, Christiansen C, Platt R. Fever in pediatric primary care: occurrence, management and outcomes. Pediatrics 2000; 105: 260-6.

8.- Tan T. Pediatric invasive pneumococcal disease in the United States in the era of pneumococcal conjugate vaccines. Clin Microbiol Rev 2012; 25: 409-19.

9.- Advisory Committee on Immunization Practices. Preventing pneumococcal disease among infants and young children. Recommendations of the Advisory Committee on Immunization Practices (ACIP). Morb Mortal Wkly Rep (MMWR) Recomm Rep 2000; 49: 1-38.

10.- Potin M. Vacunas anti-neumocóccicas en población pediátrica: actualización. Rev Chilena Infectol 2014; 31: 452-6.

11.- Dagan R, Juergens C, Trammel J, Patterson S, Greenberg D, Givon-Lavi N, et al. Efficacy of 13-valent pneumococcal conjugate vaccine (PCV13) versus that of 7-valent PCV (PCV7) against nasopharyngeal colonization of antibiotic-nonsusceptible Streptococcus pneumoniae. J Infect Dis 2015; 211: 1144-53.

12.- Vigilancia de laboratorio de Streptococcus pneumoniae procedente de enfermedad invasora. Chile, 2007-2015. Boletín Instituto de Salud Pública de Chile Vol. $5 \mathrm{n}^{\circ} 7$ julio 2015.

13.- Park I, Moore M, Treanor J, Pelton S, Pilishvili T, Beall B \& Active bacterial core surveillance team. Differential effects of pneumococcal vaccines against serotypes $6 \mathrm{~A}$ and $6 \mathrm{C}$. J Infect Dis 2008; 198: 1818-22.

14.- Jakobsen H, Sigurdsson V, Sigurdardottir S, Schulz D, Jonsdottir I. Pneumococcal serotype 19F conjugate vaccine induces cross-protective immunity to serotype 19A in a murine pneumococcal pneumonia model. Infect Immun 2003; 71: 2956-9.

15.- Alpern E, Alessandrini E, Bell L, Shaw K, McGowan K. Occult bacteremia from a pediatric emergency department: current prevalence, time to detection, and outcome. Pediatrics 2000; 106: 505-11.

16.- Pantell R, Newman T, Bernzweig J, Bergman D, Takayama J, Segal M, et al. Management and outcomes of care of fever in early infancy. JAMA 2004; 291: 1203-12.
17.- Lagos R, Muñoz A, Valenzuela M, Heitmann I, Levine M. Population-based surveillance for hospitalized and ambulatory pediatric invasive pneumococcal disease in Santiago, Chile. Pediatr Infect Dis J 2002; 21: 1115-23.

18.- Valenzuela M T, O Louglin R, de la Hoz F, Gómez E, Constenla D, Sinha A, et al. The burden of pneumococcal disease among Latin American and Caribbean children: review of the evidence. Rev Panam Salud Publica 2009; 25 (3): 270-9.

19.- Kanashiro T, Vilas Boas L, Thomaz A, Tozetto-Mendoza T, Setsuko M, Machado C. Identification of respiratory virus in infants with congenital heart disease by comparison of different methods. Rev Inst Med Trop Sao Paulo 2011; 53: 241-6.

20.- Gharabaghi F, Hawan A, Drews S J, Richardson S E. Evaluation of multiple commercial molecular and conventional diagnostic assays for the detection of respiratory viruses in children. Clin Microbiol Infect 2011; 17: 1900-6.

21.- Mahony J, Chong S, Merante F, Yaghoubian S, Sinha T, Lisle C, et al. Development of a respiratory virus panel test for detection of twenty human respiratory viruses by use of multiplex PCR and a fluid microbead-based assay. J Clin Microbiol 2007; 45: 2965-70.

22.- Mahony J. Detection of respiratory viruses by molecular methods. Clin Microbiol Rev 2008; 21: 716-47.

23.- Mahony J, Blackhouse G, Babwah J, Smieja M, Buracond S, Chong S, et al. Cost analysis of multiplex PCR testing for diagnosing respiratory virus infections. J Clin Microbiol 2009; 47: 2812-7.

24.- Torres G, Vicente M. Detection of respiratory 
syncytial and adenovirus in nasopharyngeal aspirates: comparison of cellular cultures and inmunofluorescence Rev Med Chile 1992; 120 : 415-9.

25.- Gómez R, Mora J, Cortés C, Riedel C, Ferres M, Bueno S, et al. Respiratory syncytial virus detection in cells and clinical samples by using three new monoclonal antibodies. J Med Virol 2013; 86: 1256-66.

26.- American Academy of Pediatrics, Subcommittee on Urinary Tract Infection, Steering Committee on Quality Improvement and Management; Roberts KB. Urinary tract infection: Clinical practice guideline for diagnosis and management of the initial UTI in febrile infants and children 2 to 24 months. Pediatrics 2011; 128: 595-610.

27.- Herz A M, Greenhow T L, Alcantara J, Hansen J, Baxter R P, Black S B, et al. Changing epidemiology of outpatient bacteremia in 3- to 36-month-old children after the introduction of the heptavalent-conjugated pneumococcal vaccine. Pediatr Infect Dis J 2006; 25: 293-300.

28.- Carstairs K, Tanen D, Johnson A, Kailes S, Riffenburgh R. Pneumococcal bacteremia in febrile infants presenting to the Emergency Department before and after the introduction of the heptavalent pneumococcal vaccine. Ann Emerg Med 2007: 49: 772-7.

29.- Kaplan S, Barson W, Lin P, Stovall S, Bradley J, Tan T, et al. Serotype 19A is the most common serotype causing invasive pneumococcal infections in children. Pediatrics 2010; 125: 429-36.

30.- Pilishvili T, Lexau C, Farley M, Hadler J, Harrison L, Bennett N, et al. Sustained reductions in invasive pneumococcal disease in the era of conjugate vaccine. J Infect Dis 2010; 201: 32-41.

31.- Publication, W. H. O. Pneumococcal vaccines WHO position paper-2012-Recommendations. Vaccine 2012; 30: 4717-8.
32.- Kaplan S, Barson W, Lin P, Romero J, Bradley J, Tan T, et al. Early trends for invasive pneumococcal infections in children after the introduction of the 13-valent pneumococcal conjugate vaccine. Pediat Infect Dis J 2013; 32: 203-7.

33.- Shouval D, Greenberg D, Givon-Lavi N, Porat N, Dagan R. Serotype coverage of invasive and mucosal pneumococcal disease in Israeli children younger than 3 years by various pneumococcal conjugate vaccines. Pediatr Infect Dis J 2009; 28: 277-82.

34.- Simell B, Auranen K, Käyhty H, Goldblatt D, Dagan R, O'Brien K. Pneumococcal carriage group. The fundamental link between pneumococcal carriage and disease. Exp Rev Vaccine 2012; 11: 841-55.

35.- Hammitt L, Bruden D, Butler J, Baggett H, Hurlburt D, Reasonover A, et al. Indirect effect of conjugate vaccine on adult carriage of Streptococcus pneumoniae: an explanation of trends in invasive pneumococcal disease. J Infect Dis 2006; 193: 1487-94.

36.- Spijkerman J, van Gils E J, Veenhoven R H, Hak E, Yzerman E, van der Ende A, et al. Carriage of Streptococcus pneumoniae 3 years after start of vaccination program, the Netherlands. Emerg Infect Dis 2011; 17: 584-91.

37.- Waight P, Andrews N, Ladhani S, Sheppard C, Slack M, Miller E. Effect of the 13-valent pneumococcal conjugate vaccine on invasive pneumococcal disease in England and Wales 4 years after its introduction: an observational cohort study. Lancet Infect Dis 2015; 15: 535-43.

38.- Dagan R, Patterson S, Juergens C, Greenberg D, Givon-Lav N, Porat N, et al. Comparative immunogenicity and efficacy of 13-valent and 7-valent pneumococcal conjugate vaccines in reducing nasopharyngeal colonization: a randomized double-blind trial. Clin Infect Dis
2013; 57: 952-62.

39.- Ben-Shimo S, Givon-Lavi N, Leibovitz E, Raiz S, Greenberg D, Dagan R. Nearelimination of otitis media caused by 13 -valent pneumococcal conjugate vaccine (PCV) serotypes in southern Israel shortly after sequential introduction of 7-valent/13-valent PCV. Clin Infect Dis 2014; 59: 1724-32.

40.- Zuccotti G, Mameli C, Daprai L, Garlaschi M L, Dilillo D, Bedogni G,et al Serotype distribution and antimicrobial susceptibilities of nasopharyngeal isolates of Streptococcus pneumoniae from healthy children in the 13-valent pneumococcal conjugate vaccine era. Vaccine 2014; 32 : 527-34.

41.- Hammitt L, Akech D, Morpeth S, Karani A, Kihuha N, Nyongesa S, et al. Population effect of 10 -valent pneumococcal conjugate vaccine on nasopharyngeal carriage of Streptococcus pneumoniae and non-typeable Haemophilus influenzae in Kilifi, Kenya: findings from cross-sectional carriage studies. Lancet Global Health. 2014; 2, e397-e405.Estudio COMPAS.

42.- Use of 13-valent pneumococcal conjugate vaccine and 23 -valent pneumococcal polysaccharide vaccine for adults with immunocompromising conditions:

Recommendations of the Advisory Committee on Immunization Practices (ACIP) Disponible en: http://www.cdc.gov/mmwr/preview/ mmwrhtml/mm6140a4.htm [Consultado el 11 de diciembre de 2015]. October 12, 2012/61; 816-19.

43.- Moore M, Link-Gelles R, Schaffner W, Lynfield R, Lexau C, Bennett N, et al. Effect of use of 13-valent pneumococcal conjugate vaccine in children on invasive pneumococcal disease in children and adults in the USA: analysis of multisite, populationbased surveillance. Lancet Infect Dis 2015; 15 : 301-9. 